\title{
AMON: transition to real-time operations
}

\author{
Gordana Tešić ${ }^{*}$ and Azadeh Keivani for the AMON team ${ }^{\dagger}$ \\ The Pennsylvania State University \\ E-mail: gut10@psu.edu, keivani@psu.edu
}

The Astrophysical Multimessenger Observatory Network (AMON) will link the world's leading high-energy neutrino, cosmic-ray, gamma-ray and gravitational wave observatories by performing real-time coincidence searches for multimessenger sources from observatory subthreshold data streams. The resulting coincidences will be distributed to interested parties in the form of electronic alerts for real-time follow-up observation. We will present the science case, design elements, current and projected partner observatories, and status of the AMON project. Observatories that already signed the AMON Memorandum of Understanding ( $\mathrm{MoU}$ ) include the IceCube and ANTARES Neutrino Observatories, the HAWC and VERITAS gamma-ray observatories, the Pierre Auger Cosmic Ray Observatory, the Swift satellite experiment and the MASTER robotic telescope network. AMON is an open network seeking new triggering and follow-up observatories, as well as collaborators interested in the scientific goals of AMON. The prototype of the AMON server has been online since August 2014 and processing archival data. Currently, we are deploying new high-uptime servers and will be ready to start issuing alerts as early as summer 2015.

PACS: 95.85.Pw, 95.85.Ry, 95.85.Sz, 98.70.Rz, 98.70.Sa, 07.05.Bx

The 34th International Cosmic Ray Conference,

30 July- 6 August, 2015

The Hague, The Netherlands

* Speaker.

${ }^{\dagger}$ The AMON team URL: http://amon.gravity.psu.edu/participants.shtml 


\section{Introduction}

Current and future high-energy observatories, representing a joint effort by thousands of scientists, aim to solve long standing questions about origins of cosmic neutrinos and cosmic rays, as well as an existence of gravitational waves. However, being first generation instruments, the current non-electromagnetic observatories have limited sensitivities for detection of cosmic sources. The number of statistically significant detections by these facilities is expected to be low (e.g., order of ten per year for the IceCube Neutrino Observatory that recently discovered cosmic neutrinos [1-3]). Data streams from these multimessenger facilities contain a large number of signals that are indistinguishable from background events. These so-called subthreshold events from any single observatory cannot be used alone to claim a statistically significant discovery. However, if these subthreshold events are found within a temporal and spatial coincidence of an event from another multimessenger data stream, then jointly these events could lead to the discovery of an astrophysical signal. Since most multimessenger sources are expected to be transient, it is essential to detect such coincidences in real-time and promptly distribute electronic alerts for follow-up observations, which will enable scientific exploitation of these sources. The Astrophysical Multimessenger Observatory Network (AMON) is designed to conduct these types of coincidence searches both in real-time and within archival data.

AMON will integrate heterogeneous data from several of the world's leading high-energy (neutrino, cosmic-ray and gamma-ray) and gravitational wave observatories into a single virtual system with combined sensitivity greater than that of any individual facility. The goal of AMON is to discover energetic astrophysical transient phenomena by using messengers of all four forces of nature. More details about the scientific motivation, the design elements and the anticipated discovery potential of AMON are summarized in Reference [4]. In this proceeding, we present the AMON network, its current status and its readiness for the real-time operations.

\section{AMON network}

AMON is constituted as a network of observatories and a system of cyberinfrastructure for joint analysis of multimessenger data and electronic alert distribution. Data shared across the network remain the property of the originating collaborations and all decisions about data analysis and publications are made by the participating collaborations. Further information about AMON data sharing policies can be found in AMON Memorandum of Understanding (MoU) [5].

The AMON network consists of triggering and follow-up observatories. The triggering observatories are sensitive to one or more messengers (neutrinos, photons, cosmic rays or gravitational waves) and typically have large fields of view (FoV), as well as high duty cycles. Ground based triggering observatories send their candidate events to AMON via direct links in real-time. Events from satellite experiments (e.g., Swift BAT and Fermi LAT) are received by utilizing the Gammaray Coordinates Network $(\mathrm{GCN})$ [6]. AMON also utilizes GCN to distribute its electronic alerts (in the VOEvent format [7]) to the follow-up observatories in real-time. The follow-up observatories are pointing telescopes situated on Earth (e.g., VERITAS, MASTERS etc.), as well as orbital telescopes (Swift XRT \& UVOT) that respond in near real-time to AMON alerts and perform 


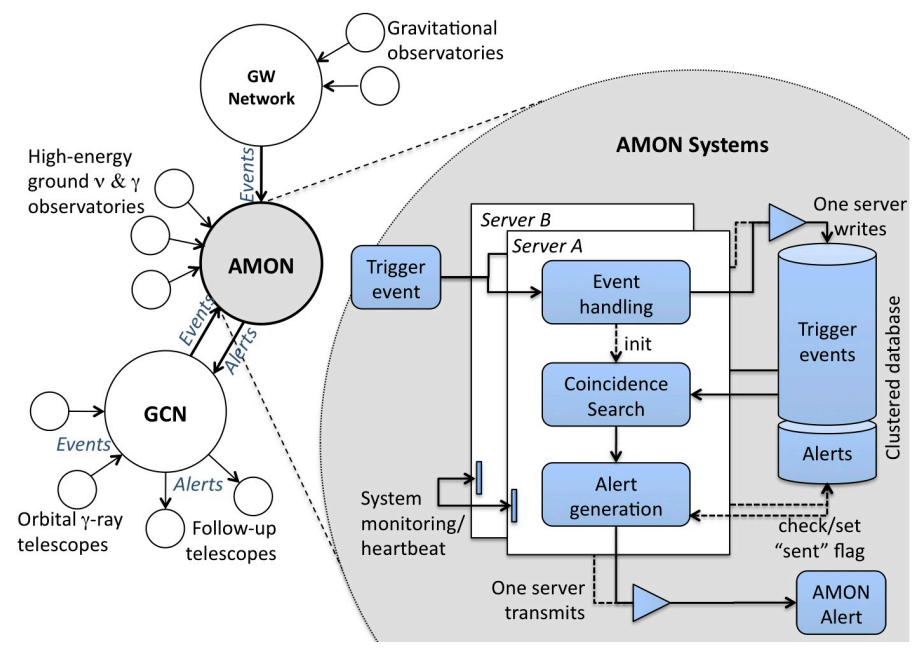

Figure 1: Data flow within the AMON network: subthreshold data from multimessenger triggering observatories (left) are sent in a standard VOEvent format and stored in a secure database (right). AMON will also receive VOEvents from satellite experiments via the Gamma-ray Coordinates Network (GCN) and will use GCN to distribute AMON alerts as VOEvents to the follow-up observatories.

follow-up observations for electromagnetic counterparts of the multimessenger signal. The flow of data within the AMON network, as well as inside the AMON system is illustrated in Figure 1.

The partner observatories that signed AMON MoU include the IceCube and ANTARES Neutrino Observatories, the HAWC and VERITAS $\gamma$-ray observatories, the Pierre Auger Cosmic Ray Observatory, the Swift satellite experiment and the MASTER robotic telescope network. Additional facilities that signed the AMON Letter of Collaboration (LoC), which is an intermediate step toward the MoU signature, are the Advanced LIGO gravitational wave detector, the Fermi LAT orbital telescope and the Palomar Transient Factory (PTF). The observatories that signed either MoU or LoC are tabulated in Table 1.

AMON is an open and extensible network seeking new collaborators that are interested in the AMON scientific goals. Currently, discussions have been initiated with the MAGIC, H.E.S.S. and FACT collaborations with the goal to bring them to the AMON network. Prospective collaborators are encouraged to contact the authors for more information about how to join the AMON network [8].

\section{Exchange protocols and data content}

Event content: Triggering facilities linked by AMON collect heterogeneous data used for detecting multimessenger coincidences. The collected data are not transmitted to AMON in their raw format, but in the higher level "trigger event" form that is specific for each observatory type. For example, a "trigger event" for IceCube represents a single particle interaction, whereas for HAWC it is constructed from the multiple photos detected above a certain threshold. For the AMON purposes, events from each observatory stream contain the following minimal information needed for the statistical analysis in the coincidence searches: a trigger time, an event position with a positional error or probability density function and a false positive rate density in the vicinity of the 


\begin{tabular}{llll}
\hline \hline Observatory & Messenger type & Role & Status \\
\hline ANTARES & neutrinos & triggering & $\mathrm{MoU}$ \\
Fermi LAT & gamma rays & triggering & $\mathrm{LoC}$ \\
Fermi GBM & gamma rays & triggering & $\mathrm{LoC}$ \\
HAWC & gamma rays & triggering & $\mathrm{MoU}$ \\
IceCube & neutrinos & triggering & $\mathrm{MoU}$ \\
LIGO & gravitational waves & triggering & $\mathrm{LoC}$ \\
LMT & radio waves & follow-up & $\mathrm{LoC}$ \\
MASTERS & optical photons & follow-up & $\mathrm{MoU}$ \\
Pierre Auger & neutrons, neutrinos and gamma rays & triggering & $\mathrm{MoU}$ \\
PTF & optical photons & follow-up & $\mathrm{LoC}$ \\
Swift BAT & X rays & triggering & $\mathrm{MoU}$ \\
Swift XRT & X rays & follow-up & $\mathrm{MoU}$ \\
Swift UVOT & UV and optical photons & follow-up & $\mathrm{MoU}$ \\
VERITAS & gamma rays & triggering/follow-up & $\mathrm{MoU}$ \\
\hline \hline
\end{tabular}

Table 1: Triggering and follow-up observatories that have signed AMON Memorandum of Understanding (MoU) or Letters of Collaboration (LoC).

event $\left(s r^{-1} s^{-1}\right)$. In addition to this event content common to each observatory, each event can have one or more parameters that are specific to each observatory stream such as: event energy (e.g., for IceCube neutrinos), signal to noise ratio (e.g., for Swift BAT triggers) or event quality based on local interactions in a given detector.

Alert content: The information from these "trigger events" from multiple streams is combined statistically by the AMON analysis algorithms that search in real-time for temporal and spatial coincidences. For each coincident signal found, AMON alert is created. Information packeted in the AMON alert includes the best fitted common source position, burst onset time, positional errors, false alarm rate, number of events and type of messengers contributing to a given alert.

Event and alert format: Trigger events from the member observatories are transmitted to AMON as XML files in the VOEvent format. AMON will also distribute its alerts about coincidences found as VOEvents. VOEvent is a standard information packet for representing, transmitting, publishing and archiving information about an astronomical (transient) event. This format was developed by the International Virtual Observatory Alliance (IVOA) [9]. Utilizing a standardized data format, such as VOEvent, simplifies protocols for data handling and distribution of alerts. For example, adding each new observatory into a follow-up and/or exchange of data program will not require new methods for injection of data into the database and analysis stream. Also new methods are not required for insertion of alert content into the alerts to be transmitted. Not less importantly, VOEvent has been used by a larger astronomical community and become a standard for real-time event distribution (e.g. GCN notices, Swift, Fermi, LIGO etc.). It is well structured in XML format with a simple schema that is easily interpreted by software and can be read by robotic telescopes. This is important both for real-time analysis, and for near real-time follow-up of the alerts. 


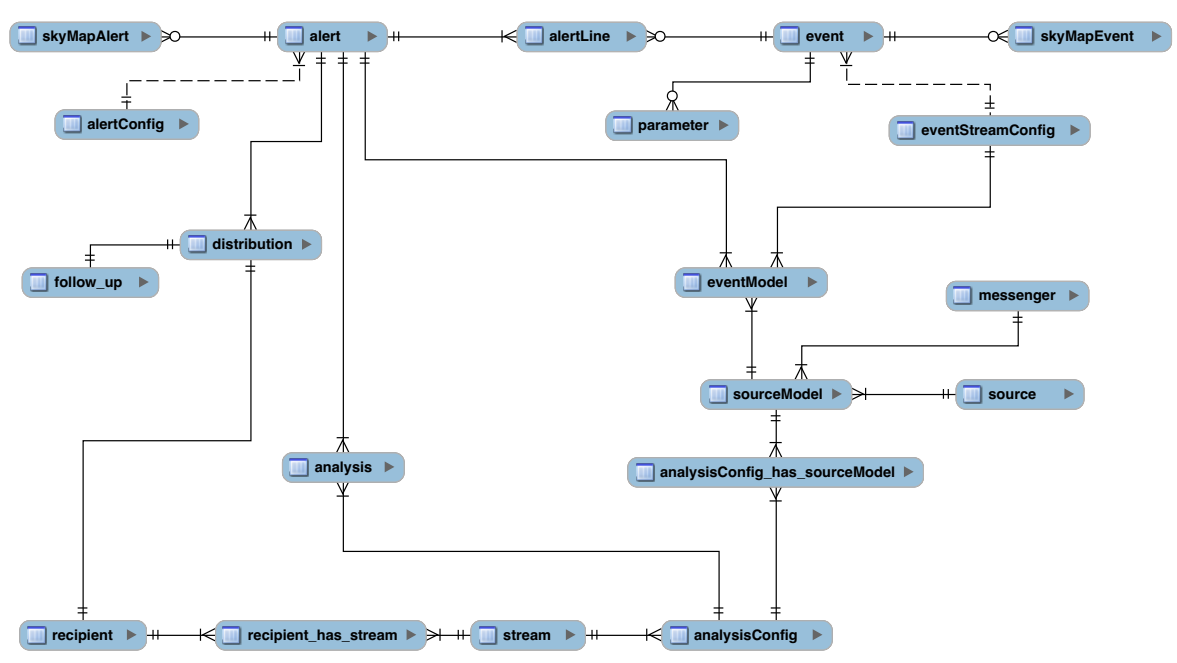

Figure 2: Relational schema of the AMON database. The database stores information from: (a) data streams of each observatory (tables "event", "parameter" and "skymap"), (b) AMON alerts ("alert" and "skymap"), (c) observatory configuration models ("eventStreamConfig"), (d) criteria used in alert search algorithms ("alertConfig"), (e) multiple analysis streams configurations including astrophysical source models ("analysis", "eventModel", "source", "sourceModel", and "messenger"), (f) a track of distribution of alerts to various recipients ("distribution", "recipient" and "stream"), and (g) information from follow-up observations of the AMON alerts ("follow-up"). Many-to-many relations between some tables are handled via appropriately defined junction tables ("alertLine", "analysisConfig_has_sourceModel” and "recipient_has_stream").

\section{The network status}

In this section, major activities within the network that lead toward full real-time operations of AMON are reviewed.

\subsection{AMON database}

The AMON system implements the world's first terabyte scale multimessenger database. The database is designed to store events received in real-time from the AMON member observatories as well as their archival data. In addition, the database stores: AMON alerts, observatory configuration models, selection criteria used in alert search algorithms, multiple analysis stream configurations including astrophysical sources, and information from follow-up observations of the AMON alerts. The schema of the AMON database is shown in Figure 2. The database underwent successful beta testing by using multiple observatory simulated, and more recently real data (blinded), both using archival data, as well as using data in real-time.

Currently, the database stores public data from: IceCube-40 and IceCube-59 strings configurations (neutrinos), Fermi LAT (gamma rays) and Swift BAT (hard X rays). We have also injected private archival data from Pierre Auger (cosmic rays) and ANTARES 2008 (neutrinos). In addition, we obtained public data from LIGO runs S5 and S6 and are in a process of writing them into the database. Finally, there is an ongoing effort to get permission from the AMON member observatories to obtain their more recent private data. 


\subsection{Archival analysis}

Under rules of AMON MoU, the participating AMON members are able to utilize the AMON database and software in order to perform both archival and real-time searches for new astrophysical signals. During the last year, the public archival data have been used by the AMON team members with the goal of developing and tuning analysis algorithms for the AMON production phase.

Within the AMON framework, we have performed a coincidence analysis of the public archival data from participating neutrino (IceCube) and gamma-ray observatories (Fermi LAT and Swift BAT) [10]. This analysis is focused on the discovery of the first jointly emitting gamma-ray and high-energy neutrino sources. We are also conducting coincidence analysis of neutrino (IceCube), gamma-ray (HAWC) and cosmic ray data (Pierre Auger), which aims to detect a distinctive primordial black hole (PBH) evaporation signature [11]. Detection of PBHs would be a scientific breakthrough confirming the Hawking's hypothesis of black hole radiation and cosmological models of phase transitions, and would allow us to probe physics at the highest energy scale. Another AMON analysis in progress searches for jointly emitting gamma-ray and gravitational wave sources by using data from Swift BAT and LIGO run S5. These types of analyses may discover first multimessenger sources or provide new constraints on jointly-emitting astrophysical source populations.

\subsection{AMON server}

The AMON real-time server has been designed, built, and tested. This is an asynchronous server written in Twisted [12], which is a Python based and event-driven networking framework. The server accepts XML messages in the form of VOEvents (Virtual Observatory Events) [7] using the HTTPS post method. This server will be receiving data from our member observatories and sending alerts to follow-up facilities when coincident signals are found. Each event received by the server is immediately written to the AMON database and sent to be processed by the dedicated AMON real-time analysis algorithm. The analysis algorithm compares each event time and arrival direction across multiple observatory streams searching for coincident events. Interesting coincidences generate AMON alerts for distribution to subscribers via GCN. The prototype of the AMON server has been online and processing archival data since August 2014. The event transmission software (known as the AMON client) is also written in Python/Twisted and uses the HTTPS Post method to send the observatory events in the VOEvent format to the AMON server. This client, as well as the entire AMON analysis software package is available to the AMON collaborators.

\subsection{Hardware}

Recently, we have procured two new high-uptime (less than 1 hour of downtime per year) servers. The physical machines are of the DELL Model PowerEdge R630 server type with memory mirroring. This system has a high level of physical and cyber security and it is both hardware and power redundant. Each physical machine is located in a separate secured data centre. Currently, we are performing stress tests of the system and expect it to be fully operational during the summer of 2015. This system is hosted and administrated by the Institute for CyberScience, which is a unit of Information Technology Services (ITS) at the Pennsylvania State University. 


\subsection{Real-time operations}

There has been significant progress toward real-time operation of AMON. In addition to the AMON server being online, we have started receiving the first event streams in real-time. In particular, there are two event streams from the IceCube observatory that are transmitted to AMON in real-time: the muon neutrino $\left(v_{\mu}\right)$ singlet stream and the high-energy starting events (HESE) stream. The $\left(v_{\mu}\right)$ stream contains subthreshold data at the rate of about $3 \mathrm{mHz}$. The HESE stream contains both above threshold (signal) and subthreshold high-energy neutrino events. Additionally, there are ongoing efforts with the member collaborators from Pierre Auger and HAWC collaborations to get their subthreshold data streams in real-time to AMON.

In May, 2015, AMON established a connection with GCN [6,13]. The GCN client directly connects to AMON by using a so-called vTCP (VOEvent over TCP) protocol and receives AMON alerts. The AMON alert streams at GCN are established as private streams that are initially available only to collaborators. In the future, any of these streams can be made public with the approval of the corresponding observatories whose events participate in that particular alert stream. As early as summer 2015, AMON will start issuing real-time alerts via GCN to its collaborators and follow-up facilities.

\section{Conclusion}

Multimessenger astroparticle physics is a field that has grown rapidly since the current generation of non-electromagnetic observatories started continuously monitoring large portions of the sky. The AMON cyberinfrastructure enables real-time and archival searches for possible multimessenger sources by conducting joint subthreshold analysis of multimessinger data. AMON has made significant progress in both real-time and archival analysis. It will start issuing electronic alerts via GCN in the summer of 2015. Jointly, the AMON partner facilities may probe the high-energy universe via all four forces of nature for the first time in human history.

\section{Acknowledgments}

This research is supported by grants from the U.S. National Science Foundation (003403953) and the Institute for Gravitation and the Cosmos of the Pennsylvania State University.

\section{References}

[1] M. G. Aartsen et al., Evidence for High-Energy Extraterrestrial Neutrinos at the IceCube Detector, Science 3421242856 (2013), [arXiv: 1311. 5238v2]

[2] M. G. Aartsen et al., Probing the origin of cosmic-rays with extremely high energy neutrinos using the IceCube Observatory, Phys. Rev. D 88112008 (2013), [arXiv: 1310. 5477v2]

[3] M. G. Aartsen et al., Observation of High-Energy Astrophysical Neutrinos in Three Years of IceCube Data, Phys. Rev. Lett. 113101101 (2014), [arXiv:1405.5303v2]

[4] M.W.E. Smith et al., The Astrophysical Multimessenger Observatory Network (AMON), Astrop. Phys. 45 56-70 (2013) [arXiv:1211.5602v1]. 
[5] The AMON team, The AMON Memorandum of Understanding, URL http://amon.gravity.psu.edu/mou_aug2012.shtml (2012).

[6] Gamma-ray Coordinates Network (GCN), URL http://gcn.gsfc.nasa.gov/

[7] International Virtual Observatory Alliance, VOEvent, URL http://www.ivoa.net/documents/VOEvent/index.html

[8] The AMON team, How to join AMON, URL http://amon.gravity.psu.edu/join.shtml

[9] International Virtual Observatory Alliance, URL http://www.ivoa.net/

[10] A. Keivani et al., AMON Searches for Jointly-Emitting Neutrino + Gamma-Ray Transients, in proceedings of ICRC, POS ( ICRC2015) 786 (2015).

[11] G. Tešić, Searching for primordial black hole evaporation signal with AMON, in proceedings of ICRC, POS ( ICRC2015) 328 (2015).

[12] Twisted project, URL https://twistedmatrix.com

[13] S. Barthelmy (the principal investigator of GCN), Private communication. 